\title{
Design and implementation DVB-S \& DVB-S2 systems
}

\author{
Samir Jasim Mohammed ${ }^{1}$, Zaid Saadi Hussein ${ }^{2}$ \\ ${ }^{1}$ Department of Electronics and Telecommunication, University of Babylon, Iraq \\ ${ }^{2}$ Department of Electronics and Telecommunication, Electrical Engineering University of Babylon, Iraq
}

\begin{tabular}{l}
\hline \hline Article Info \\
\hline Article history: \\
Received Jan 10, 2020 \\
Revised Apr 1, 2020 \\
Accepted Jun 28, 2020 \\
\hline
\end{tabular}

Keywords:

$\mathrm{BCH}$

Digital television

DVB-S

DVB-S2

LDBC

\begin{abstract}
Digital video broadcasting plays an important role in most digital communication systems. Digital broadcasting systems are designed with great accuracy and delicate design which has a major role in our lives today especially digital video broadcasting. It is these systems that are considered to be the most advanced in transmission, reception and reliability. From these systems they develop through the digital video broadcasting project group. The most important are digital video broadcasting of terrestrial, digital video broadcasting of cable and digital video broadcasting of satellite because of that many user's desires to use communications and entertainment in their lives there are new and demanding situations that the standards of these systems can only meet for their own applications .In this paper, it will design and implement the digital video broadcasting of satellite first generation and digital video broadcasting of satellite second generation of binary data,image and audio using Matlab-Simulink environment.In addition, will calculate the bit error rate of the signal. This system is designed with precision to improve performance errors and increase transmission capacity as well as provide a safe environment for information.
\end{abstract}

Copyright (C) 2020 Institute of Advanced Engineering and Science. All rights reserved.

\section{Corresponding Author:}

Zaid Saadi Hussein

Department of Electronics and Telecommunication

Electrical Engineering, University of Babylon,

Babylon, Iraq.

Email: zaidiysadd@gmail.com

\section{INTRODUCTION}

DVB (Digital Video Broadcasting) is a European institution founded in 1993, to promote international communication standards for digital TV applications. Its mission is to design and change the procedures used to convey virtual signals for information and services [1]. DVB has developed exceptional broadcast standards based on channel transmission channel :

- Terrestrial: DVB-T and DVB-T2.

- Cable: DVB-C and DVB-C2.

- Satellite: DVB-S and DVB-S2.

Each criterion defines channel coding and modulation, because each channel has a different set of properties. There are two generations of the DVB-S standard [2-6]. Standards are implemented by ETSI, especially in this case:

DVB-S:

- This was developed from 1993-1997.

- Defined by European Standard EN 300421.

- Just describes the physical link traits and the framing [7]. The overload delivery stream shipping falls beneath MPEG-2. 
DVB-S2 [8-13]:

- Was developed in 2003.

- Defined by European Standard EN 302307

- The trendy become ratified in March 2005.

- Mainly designed to deal with larger records costs to offer HDTV.Internet access and statistics distribution.

Ondřej Kaller and Ladislav Polák andTomáš Kratochvíl [14]. They have simulated and implemented features DVB-S2 and the way of regularization and the process of sending and receiving. K.C. Cinnati Loi and Seok-Bum [15]. They study the architecture of a use field-programmable gate-array (FPGA) implement of a low-density parity-check (LDPC) decoding for the digital video broadcasting second generation and appliciation. Mohammed El-Hajjar and Lajos Hanzo [16]. They study is a survey of the transmission and receiving techniquesused in digital television (TV) standards of DVB-S worldwide with the increase in the demand for high-definition (HD). Alberto Morello and vittoraia Mignone [17]. They Study properties features knowledge of specific applications of DVB-S and DVB-S2 and change of modulation of the system. Urvashi pal [18]. They study on the standardization of high DVB-S2 and its features and applications. Previous studies have studied the characteristics and features of systems of DVB-S and DVB-S2 most of the problems were that they did not design a practical system that sends video and audio and pictures as well as the rate of sending and receiving data at lower speed. In this proposed system, it will be designed a system works to transmit voice and pictures with high transmission data speed with the high data rate and faster time of the sent signal.

\section{DVB-S2 SYSTEM ARCHITECTURE}

In 2003, It was generated by the DVB Project. Then, in March 2005, ETSI ratifies it [19]. DVB-S2 Standard is considered as a major step forward compared to the DVB-S one. Figure 1 represents the DVB-S2 block diagram. It based on a Forward Error Correction technique of BCH encoder and LDPC encoder concatenated [20]. Moreover, the standard involves a wide range of modulation formats and different rates of coding. Different modulations type (QPSK, 8PSK, 16APSK, and 32APSK) are utilized in DVB-S2 [21-25]. Under the same conditions of transmission, the capacity of DVB-S2 is typically increased by $30 \%$ over DVBS. As a result, DVB-S2 operates over a SNR range exceeding 18dB; while QPSK based on DVB-S operating range is restricted to less than $5 \mathrm{~dB}[26]$.

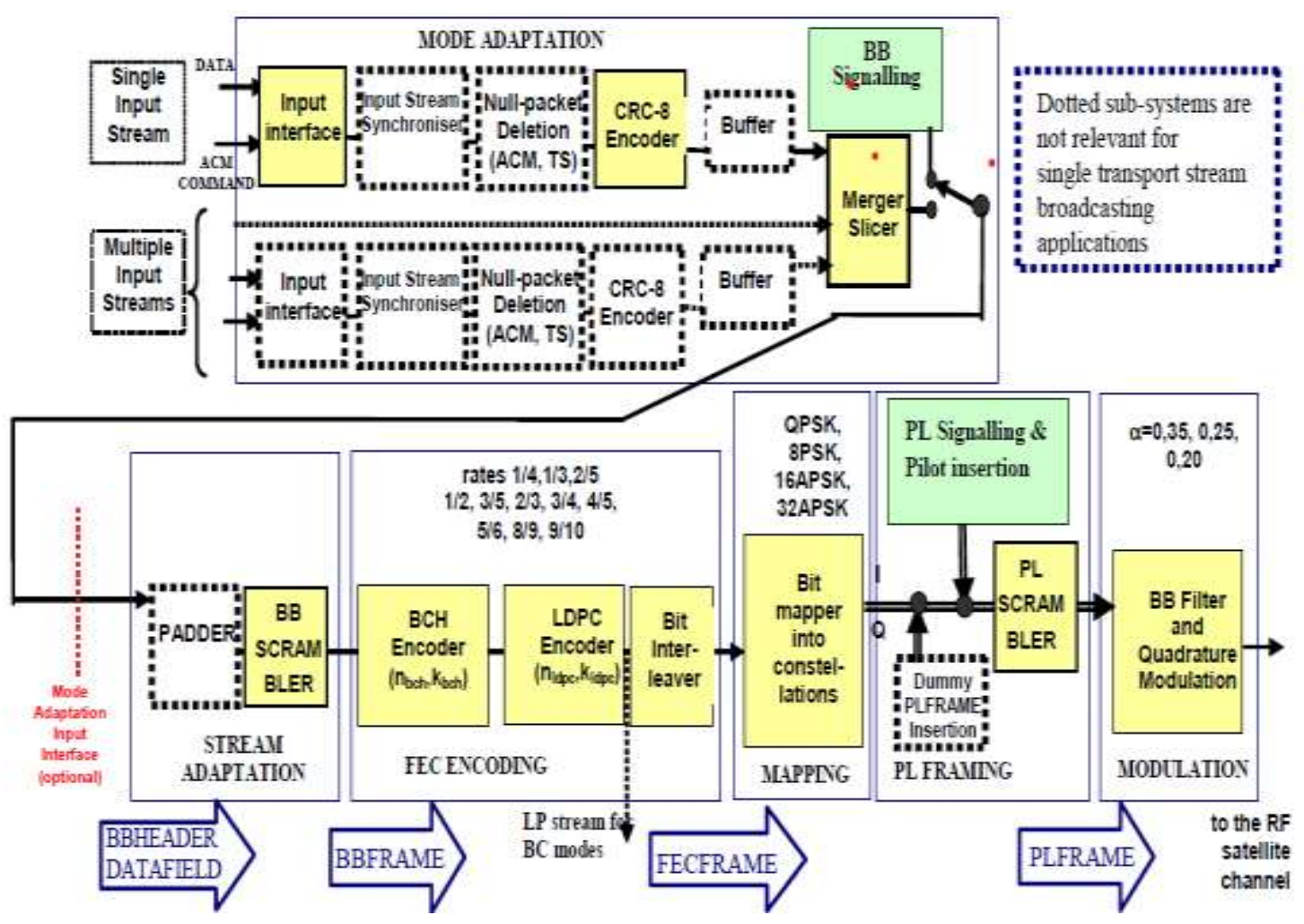

Figure 1. Functional block diagram of the DVB-S2 system 


\section{IMPLEMENTING THE DVB-S AND DVB-S2 USING MATLAB SIMULINK}

We will implement this system for several cases which will explain in the following points:

\subsection{DVB-S of binary data}

In this case, we have sent a binary signal as random integer data and then coded by Reed-Solomon encoder as outer encoder after that interleave data by convolutional interleaver as inner encoder then the signal modulated by (QPSK) modulation then transmitt signal through (AWGN) channel. The output signal from (AWGN) is demodulated by (QPSK) demodulation which deinterleavered by convolutional as inner decoder then it is decoded by Reed-Solomon as outer decoder at the last we received the data, and the parameters of block diagram are:

1. Transmitter:

- Samples time of random integer (2.61ms) \& samples per frame is (188-bit)

- Reed Solomon codeword length ( $\mathrm{N}=204$-bits) \& message length (K=188-bits)

- Convolutional interleave rows of shift register (12) \& register length steps (17)

- Trellis structure of rate punctured convolutional code poly2trellis

- Output buffer size (per channel) is (756-bits), then general block interleaves, then Output buffer size (per channel) is (9072-bits)

- DVB-S QPSK mapper

2. Channel:

- AWGN we change the SNR from (1-12) dB to calculate BER for system

3. Receiver:

- DVB-S2 QPSK Demapper.

- Viterbi decoder trellis structure poly2trellis (7, [171 133])

- Bit to integer converter number of bits per integer $(\mathrm{M})$ is (8-bits), then the convolutional deinterleaver rows of shift register (12) \&register length steps (17)

- Reed Solomon codeword length (N=204-bits) \& message length (K=188-bits)

- Number of bits per integer (8)

The circuit as shown in Figure 2.

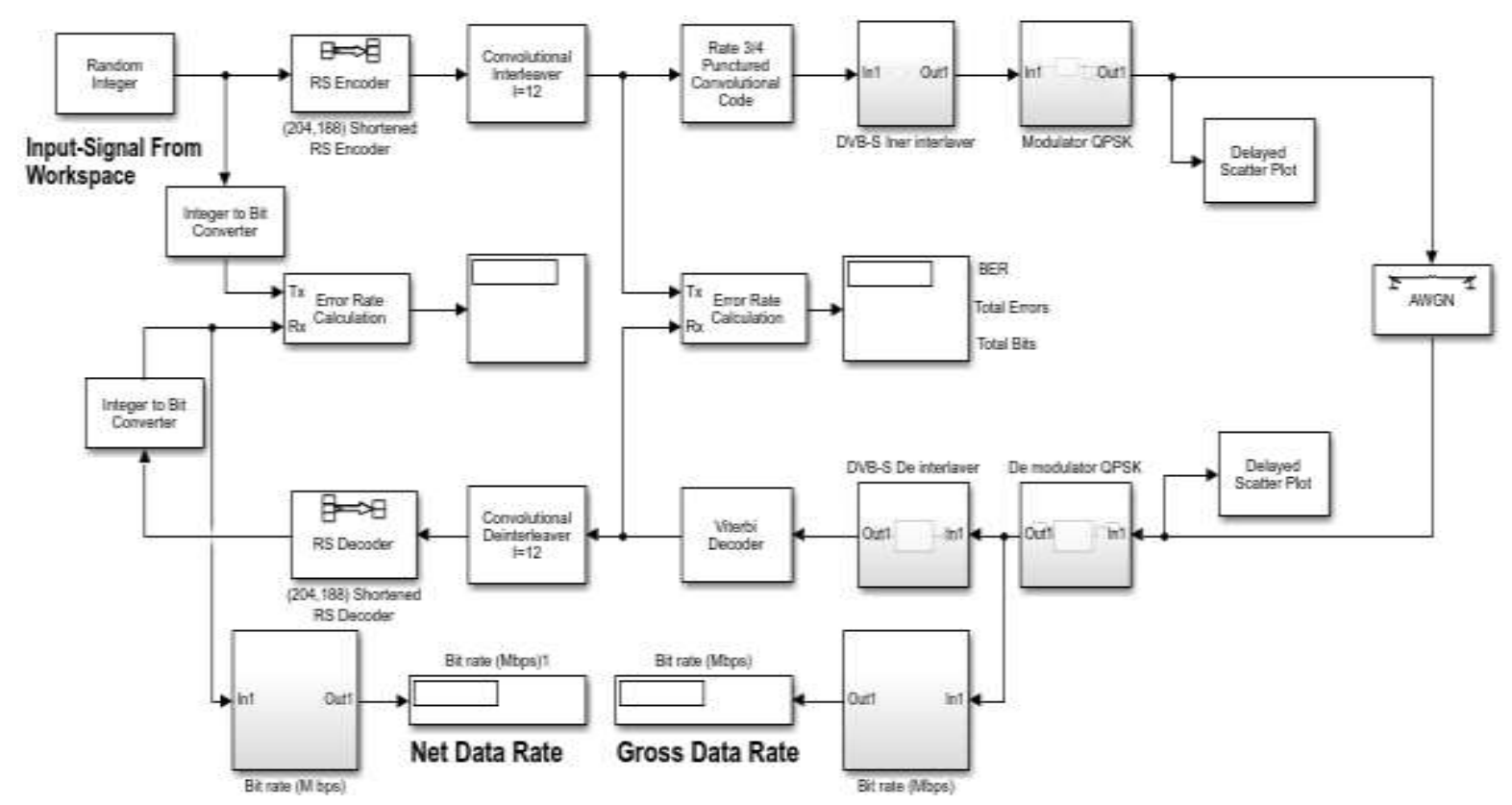

Figure 2. MATLAB-simulink of data (DVB-S)

\subsection{DVB-S2 of binary data}

In this case, we have sent a binary signal as Bernoulli binary data passes through (frame unbuffering) and then coded by $(\mathrm{BCH})$ encoder as outer encoder and (LDPC) as inner encoder the signal interleaved through 
general block interleaver then the signal modulated by (QPSK) modulation then signal transmitt through (AWGN) channel. The output signal from (AWGN) is demodulated by (QPSK) (soft-decision) demodulation which deinterleavered by general block deinterleaver then the signal decoding by (LDPC) decoder as inner decoder then it is decoded by $(\mathrm{BCH})$ as outer decoder the signal passes through (frame unbuffering) at the last we received the data, the parameters of block diagram are:

1. Transmitter:

- $\quad$ Bernoulli binary the probability of zero is (0.5) and samples time of bit (2 ms)

- Frame buffering (32208-bits)

- $\quad$ BCH encoder codeword length( $\mathrm{N}=32400$-bits) \& message length ( $\mathrm{K}=32208$-bits)

- LDPC encoder (64800-bits)

- General block interleaver

- QPSK modulator (32400-bits)

\section{Channel:}

- $\quad$ AWGN we change the SNR from (1-10) dB to calculate BER for system.

3. Receiver:

- QPSK soft decision modulator (64800-bits)

- General block deinterleaver

- $\quad$ LDPC decoder (32400-bits)

- $\quad$ BCH decoder code word length(N=32400-bits) \& message length (K=32208-bits)

- $\quad$ Bbframe unbuffering (32208-bits)

The circuit as shown in Figure 3.

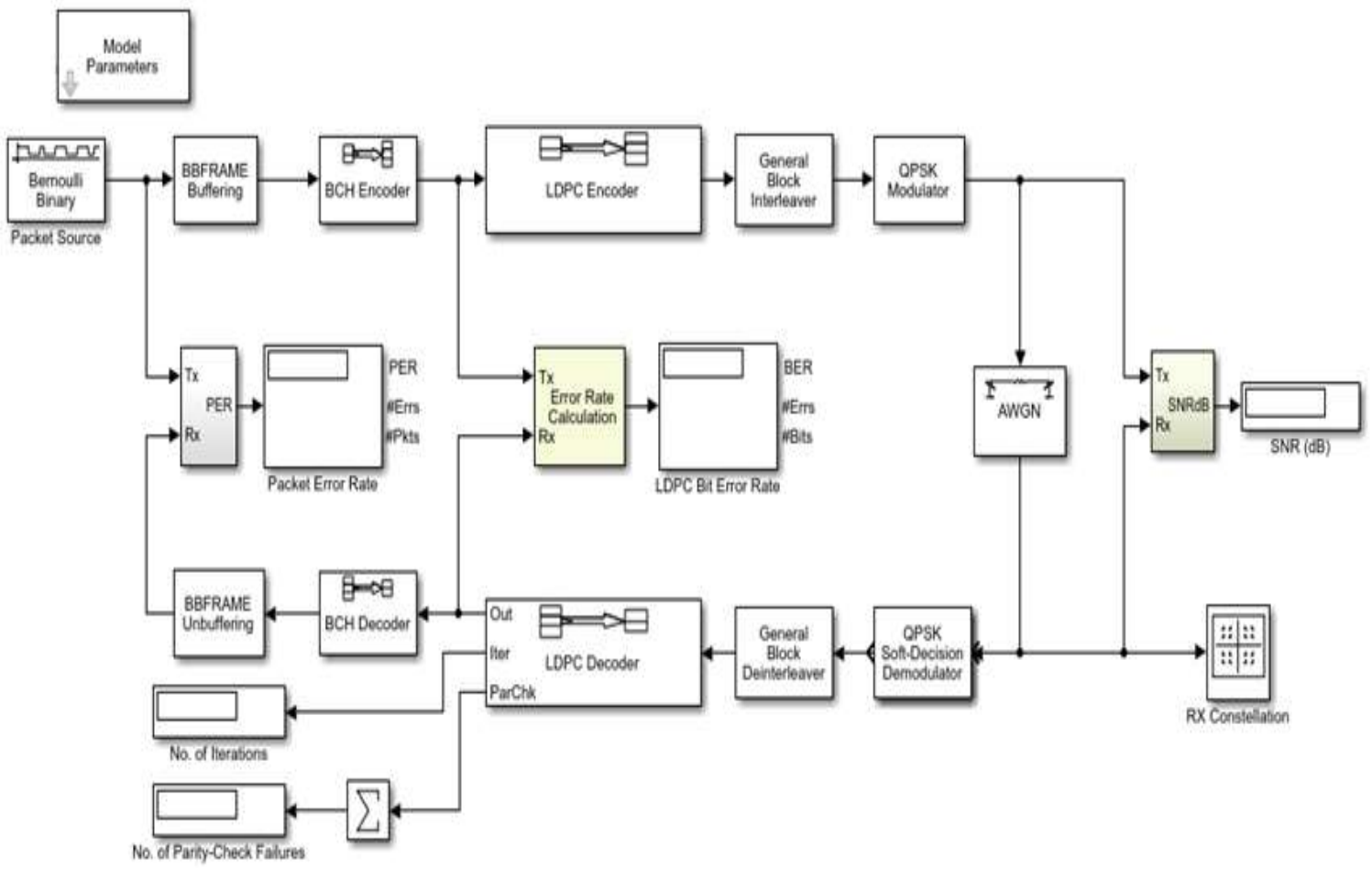

Figure 3. MATLAB-simulink of data (DVB-S2)

\subsection{DVB-S2 of sound}

In this case, we have sent a sound signal as $\mathrm{MBC}$ wave $\mathrm{A}=22050 \mathrm{HZ} 16$ bit audio mono passes through (frame buffering) and then coded by $(\mathrm{BCH})$ encoder as outer encoder and (LDPC) as inner encoder the signal interleaved through general block interleave then the signal modulated by (QPSK) modulation than signal transmitt through (AWGN) channel. The output signal from (AWGN) is demodulated by (QPSK) (soft decision) demodulation which deinterleavered by general block deinterleaver then the signal decoding by (LDPC) decoder as inner decoder then it is decoded by $(\mathrm{BCH})$ as outer decoder the signal passes through (frame unbuffering)at the last we received the sound the parameters of block diagram are: 
1. Transmitter:

- $\quad$ Sound signal as MBC wave, A=22050 HZ, 16-bit Audio buffering (32208-bits)

- $\quad$ BCH encoder codeword length( $\mathrm{N}=32400$-bits) \& message length (K=32208-bits)

- LDPC encoder (64800-bits)

- General block interleaver

- QPSK modulator (32400-bits)

2. Channel:

- $\quad$ AWGN we change the SNR from (1-10) dB to calculate BER for system.

3. Receiver:

- QPSK soft decision modulator (64800-bits)

- General block deinterleaver

- LDPC decoder (32400-bits)

- $\quad$ BCH decoder code word length(N=32400-bits) \& message length (K=32208-bits)

- Frame unbuffering (32208-bits)

The circuit as shown in Figure 4.

\section{DVB-S.2 Link, Including sound}

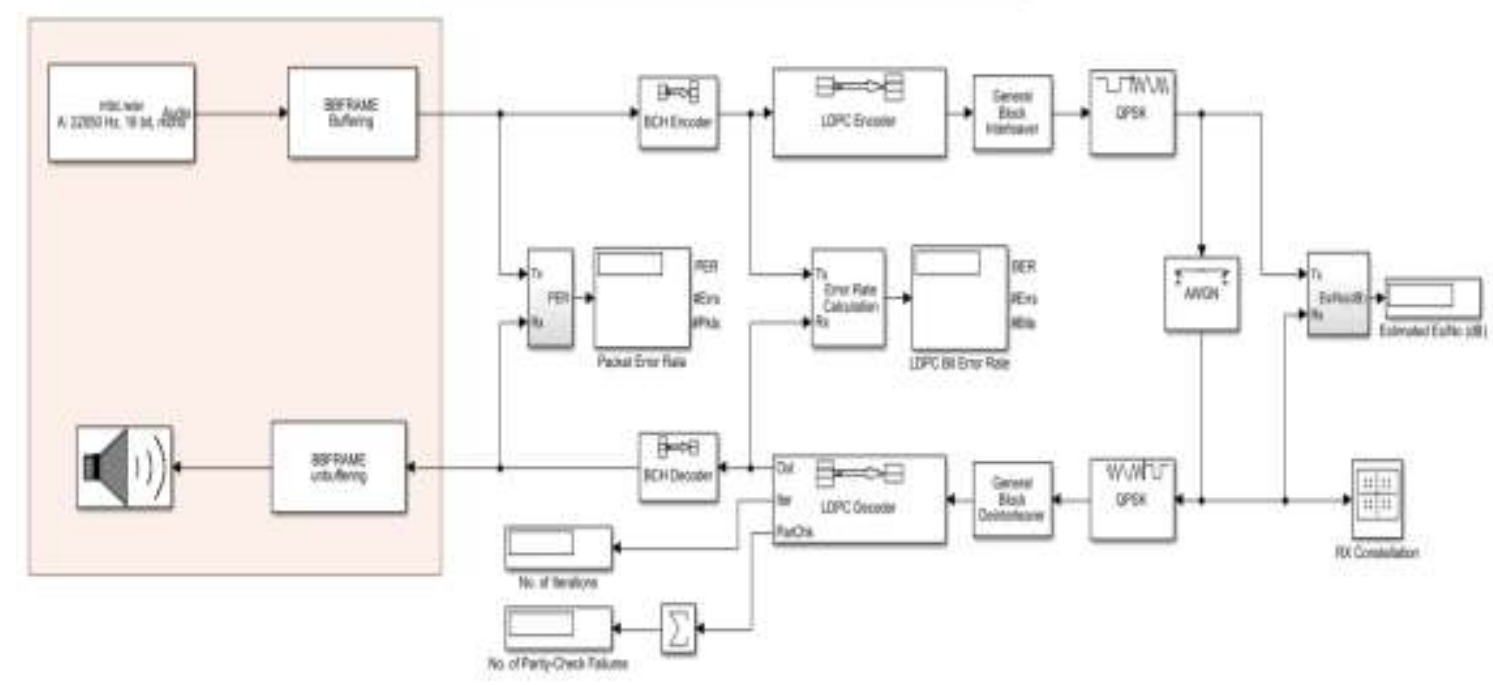

Figure 4. MATLAB simulink DVB-S2 of sound

\subsection{DVB-S2 of image}

We sent an image with dimensions of $(320 * 500 * 3)$ which passes through buffer after that the image enter to the $\mathrm{BCH}$ encoder which consider as outer encoder and (LDPC) as inner encoder the signal interleaved through general block interleave then the signal modulated by (QPSK) modulation then image transmit through (AWGN) channel.The output signal from (AWGN) is demodulated by (QPSK) demodulation which deinterleavered by general block deinterleaver, then the signal decoding by (LDPC) decoder as inner decoder then it is decoded by $(\mathrm{BCH})$ as outer decoder the signal passes through unbuffering at the last we received the image, the parameters of block diagram are:

1. Transmitter:

- Image with dimensions of $(320 * 500 * 3)$

- $\quad$ BCH encoder codeword length( $\mathrm{N}=32400$-bits $) \&$ message length ( $\mathrm{K}=32208$-bits)

- LDPC encoder (64800-bits)

- General block interleaver

- QPSK modulator (32400-bits)

2. Channel:

- AWGN we change the SNR (1-10) to calculate BER for system

3. Receiver:

- QPSK modulator (64800-bits) 
- General block deinterleaver

- $\quad$ LDPC decoder (32400-bits)

- $\quad$ BCH decoder code word length(N=32400-bits) \& message length (K=32208-bits)

The circuit as shown in Figure 5

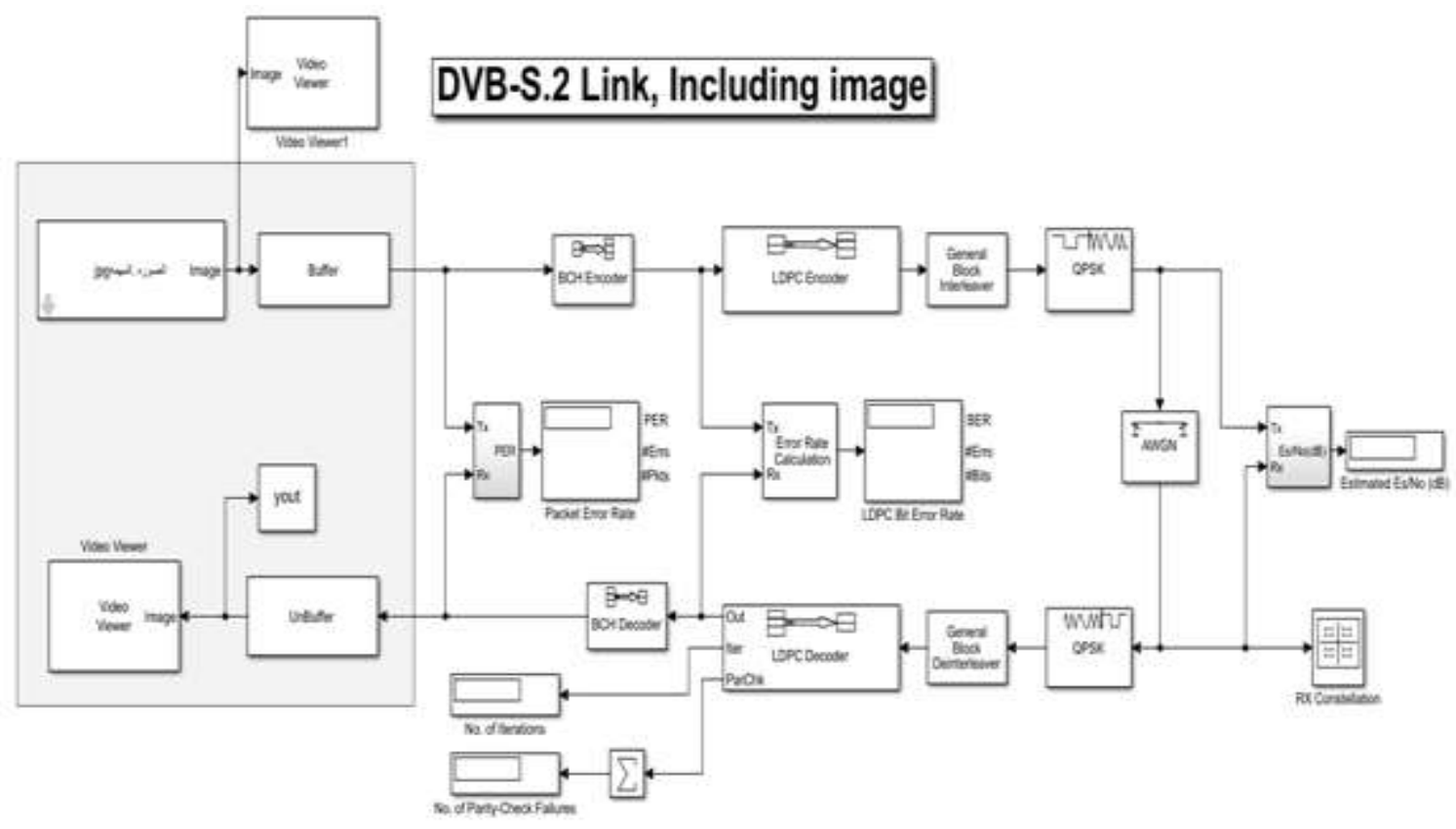

Figure 5. MATLAB simulink DVB-S2 of image

\section{RESULTS AND DISCUSSION}

This section will discuss the results that obtain from designed DVB-S of data, DVB-S2 of data, DVBS2 of sound and DVB-S2 of image.

\subsection{DVB-S of data}

After discussing the design of this system as in the parameters that was mentioned earlier. The obtained results represent the bit error rate with a change signal to noise ratio (SNR) as shown in the curve below, i.e. in the Figure 6. And note from the curve when increasing (SNR)it will decrease probability of bit error rate in the signal compared to other studies few error rates were obtained unlike the researchers the signal errors received were higher.

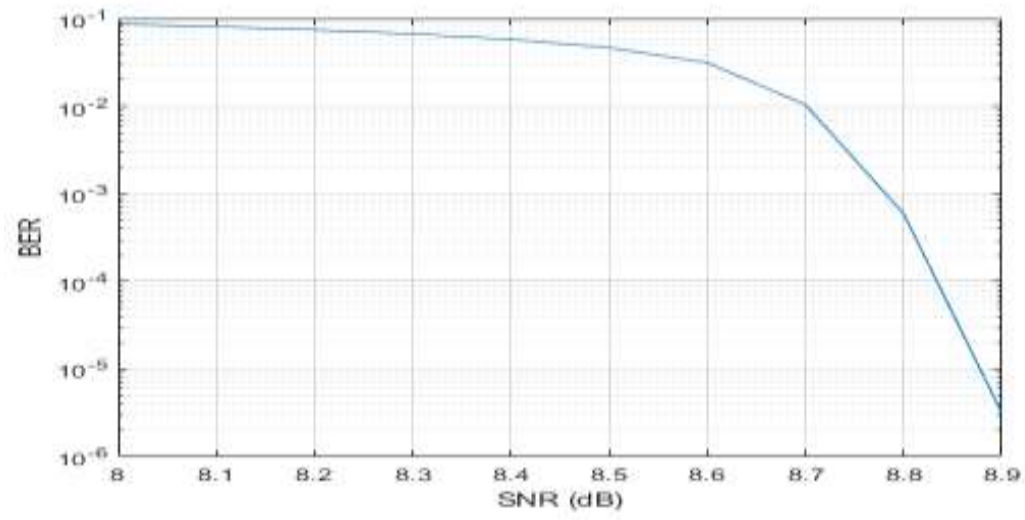

Figure 6. SNR \$ BER DVB-S OF DATA 


\subsection{DVB-S2 of data}

The results were obtained after sent the signal and as indicated by its parameter in the previous circuit as the following figures, i.e. The Figure 7 (a), Figure 7 (b), restpectively and also represents a change between the signal to noise ratio and probability bit error rate. It will be seen as in the following figures received constellation. In the results and the shown figures that have appeared and found that since we increase the signal-to-noise ratio (SNR), the signal reception will be more accurate in clarity as well as a lower error rate. And when you reduce SNR the error rate will increase in the received signal.

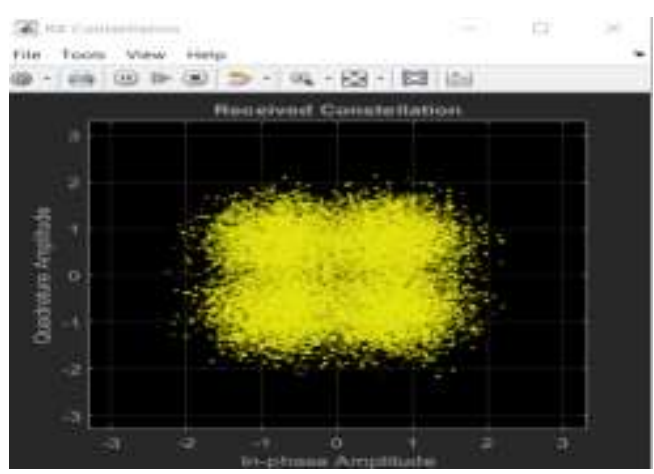

(a)

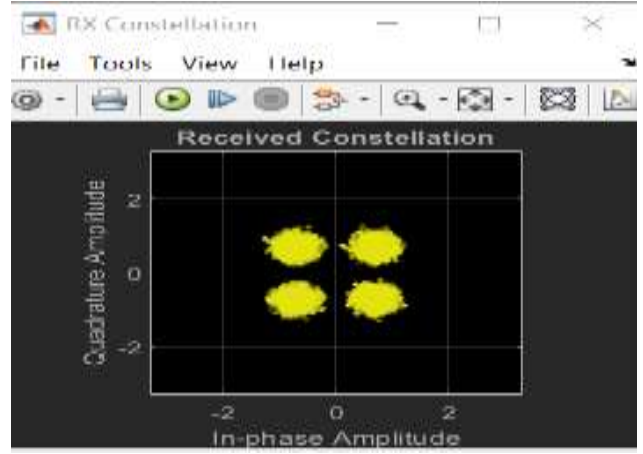

(b)

Figure 7. (a) Received constellation when SNR equal 4 dB, (b) Received constellation when SNR equal 8 dB

\subsection{DVB-S2 of sound}

Given the design and analysis of previous studies they did not mention the transmission of sound because of the problems related to modulation and coding and decoding but we touched upon it and sent the voice with accuracy with a change SNR as shown in the following Figures 8 (a) and (b). The audio parameters that have been sent are shown in the circuit that was previously addressed.

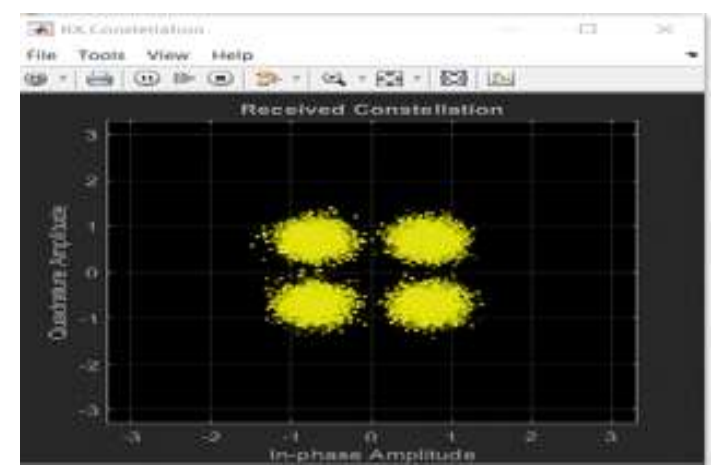

(a)

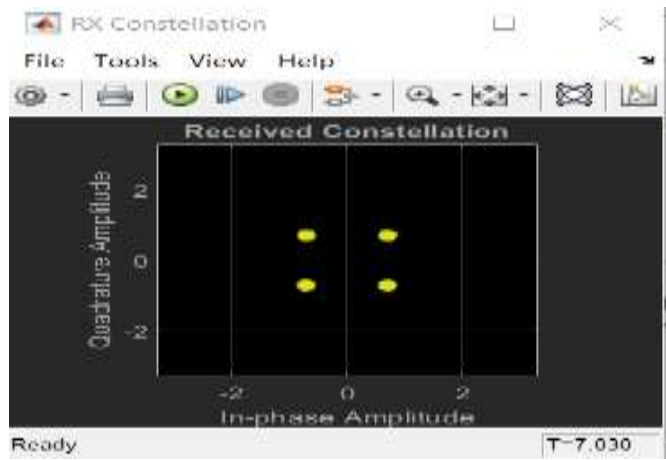

(b)

Figure 8. (a) Received constellation when SNR equal 6 dB. (b) Received constellation when SNR equal $12 \mathrm{~dB}$

\subsection{DVB-S2 of image}

Parameters related to the transmission of images were previously discussed and this design is considered a major development in communications systems such as DVB-S2. Figure 9(a) represent the transmitter of the image, Figure 9(b) represent the received image, Figure 9(c) represent a receiving constellation when SNR is $5 \mathrm{~dB}$, Figure 9(d) represent a receiving constellation when it is equal to SNR equal $10 \mathrm{~dB}$. Where the increase SNR the bit error rate will decrease in the received image as the bit error rate percentage bitapproaches to (0.0002) when SNR equal $12 \mathrm{~dB}$ and bit error rate equal (0.162) when use power signal SNR equal $-5 \mathrm{~dB}$.

The results were clear and accurate to suit the practical reality of implementing this system. In comparison with previous studies, practical and accurate results were obtained and this has not been proven by previous studies. Whereas the BER less when increases SNR of the signal and vice versa. 


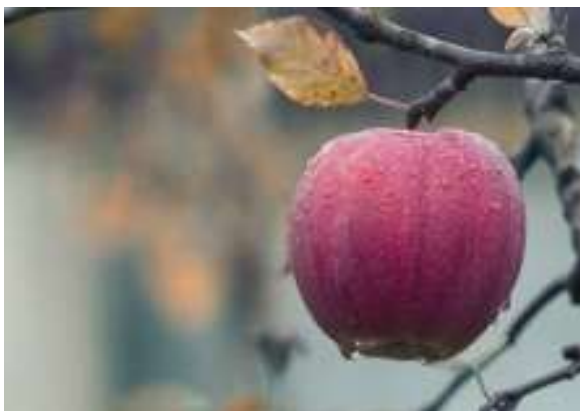

(a)

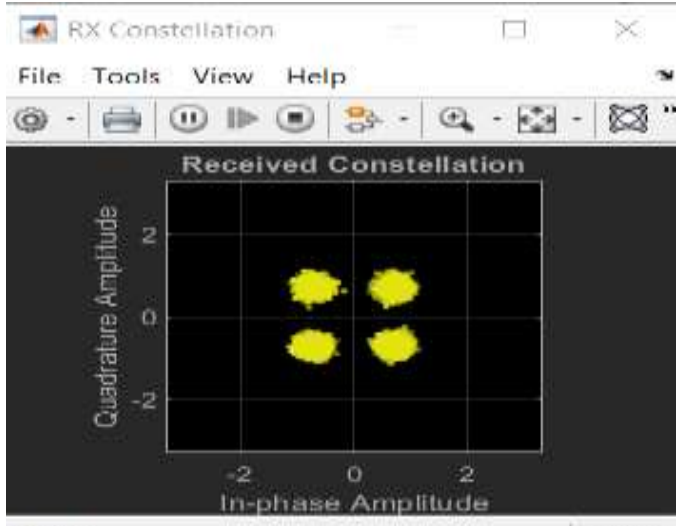

Running

(c)

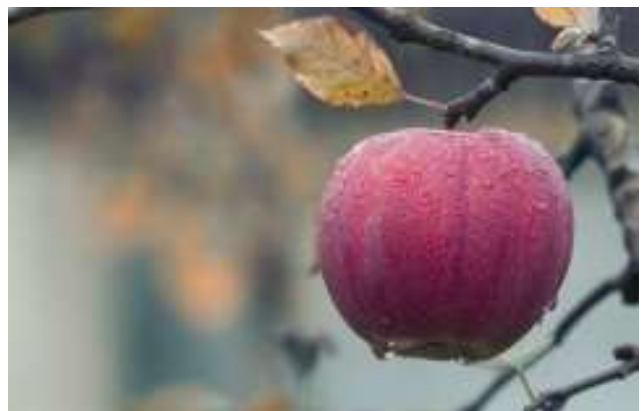

(b)

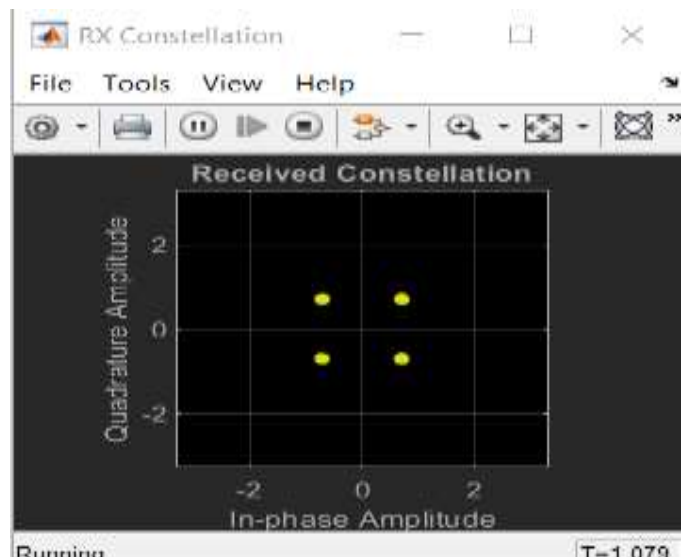

(d)

Figure 9. (a) Transmitted image. (b) Received image. (c) Received constellation when SNR equal 5 dB. (d)

Received constellation when SNR equal $10 \mathrm{~dB}$

\section{CONCLUSION}

The work recommended in this article, which includes designing and implementing DVB-S and DVB-S2 in order to know the defects and features related to these systems so that they are avoided by subsequent researchers. Where the binary data, voice and image were sent. The results were clear with regard to the probability of error and the received constellation. The SNR of the transmitter signal was changed to obtain a small number of BER and the accuracy of the received signal. The future work is to send the video in these systems, as well as the implementation of these systems through the FPGA program.

\section{REFERENCES}

[1] N. Kakanakov, D. G. Grozev, and B. Ribov, "Digital video broadcasting satellite receiver based on high," ELECTRONICS, 2005.

[2] S. Gupta, Ritika, and Sanjeev, "Digital video broadcasting (DVB): Architecture, applications, benefits and design," Int. J. Core Eng. Manag., vol. 2, no. 5, pp. 8-17, 2015.

[3] W. Stallings, Pearson new international edition wireless communications and networks. 2014.'

[4] D. J. C. Shu Lin, "Shu Lin, Daniel J. Costello-Error Control Coding (2nd Edition)-Prentice Hall (2004).pdf.” 2004.

[5] E. Feltrin, E. Weller, E. Martin, and K. Zamani, "Design, implementation and performances analysis of an on board processor - Based satellite network," IEEE Int. Conf. Commun., vol. 6, no. c, pp. 3321-3325, 2004.

[6] A. Thesis, "MATLAB Simulation of DVB-T Codec System," 2009 19th International Conference Radioelektronika, pp. 315-318, 2009.

[7] European Telecommunications Standards Institute, "EN 302 307-1 - V1.4.1 - Digital Video Broadcasting (DVB); Second generation framing structure, channel coding and modulation systems for Broadcasting, Interactive Services, News Gathering and other broadband satellite applications; Part 1: DVB-S2," En 302 307-1 V1.4.1, vol. 1, pp. 1-80, 2014.

[8] K. El-Abbasy, B. Abdelhamid, and S. Elramly, "Performance evaluation of DVB-S2 and DVB-S2X systems," 2015 IEEE International Conference on Communication, Networks and Satellite (COMNESTAT), pp. 115-120, 2016

[9] ETSI, "Digital Video Broadcasting (DVB): Framing structure, channel coding and modulation for 11/12 GHz satellite services," En 300 421, vol. 1.1.2, pp. 1-24, 1997, doi: EN 300421 V1.1.2. 
[10] R. U. Sudarwan and Iskandar, "Performance analysis of DVB-H over high altitude platform station system (HAPS)," 2015 1st Int. Conf. Wirel. Telemat. ICWT 2015, pp. 2-6, 2016.

[11] R. Shrestha and R. Paily, "Performance and throughput analysis of turbo decoder for the physical layer of digitalvideo-broadcasting-satellite-services-tohandhelds standard," IET Commun., vol. 7, no. 12, pp. 1211-1220, 2013.

[12] L. Rysdale, P. De Bot, and S. N. Hulyalkar, "Digital video broadcasting: Satellite specification,” Philips J. Res., vol. 50, no. 1-2, pp. 91-104, 1996.

[13] R. De Gaudenzi, A. Guillén i Fàbregas, and A. Martinez, "Performance analysis of turbo-coded APSK modulations over nonlinear satellite channels," IEEE Trans. Wirel. Commun., vol. 5, no. 9, pp. 2396-2407, 2006.

[14] B. Lábsky and T. Kratochvíl, "DVB-S/S2 satellite television broadcasting measurement and comparison," 20th International Conference Radioelektronika 2010, pp. 61-64, 2010.

[15] K. C. C. Loi and S. B. Ko, "Improvements on the design and implementation of DVB-S2 LDPC decoders," Comput. Electr. Eng., vol. 37, no. 6, pp. 1137-1146, 2011.

[16] M. El-Hajjar and L. Hanzo, "A survey of digital television broadcast transmission techniques," IEEE Commun. Surv. Tutorials, vol. 15, no. 4, pp. 1924-1949, 2013.

[17] A. Morello and V. Mignone, "DVB-S2: The second generation standard for satellite broad-band services," Proc. IEEE, vol. 94, no. 1, pp. 210-226, 2006.

[18] U. Pal, "On The Standardization of Ultra-High-Definition ( UHD ) Video Transmission by Digital Video Broadcasting - Satellite Second Generation ( DVB-S2 )," Ph.D. dissertation, 2016.

[19] ETSI EN 300 744, "Digital Video Broadcasting (DVB); Framing structure, channel coding and modulation for digital terrestrial television," vol. 1.6.2, 2015.

[20] L. I. Lundstrom, 'Understanding digital television: an introduction to DVB systems with satellite, cable, broadband and terrestrial TV distribution'. Routledge, 2012.

[21] B. Azarbad and A. Binti, "DVB-S2 Model in Matlab: Issues and Impairments," MATLAB - A Fundam. Tool Sci. Comput. Eng. Appl., vol. 2, pp. 217-234, 2012.

[22] P. Specification, DVB-S.2 FEC Encoder v1.3, 2007.

[23] A. B. Biran, What every engineer should know about MATLAB® and simulink ${ }^{\circledR}, 2010$.

[24] R. De Gaudenzi, A. Guillén i Fàbregas, A. Martinez, G. Colavolpe, G. Gallinaro, and N. Alagha, "Performance analysis of a mesh satellite system based on linear and continuous phase modulations," IEEE Int. Conf. Commun., pp. 3255-3259, 2012.

[25] O. K. Hamid, R. B. Abduljabbar, and N. J. Alhyani, "Fast and robust approach for data security in communication channel using pascal matrix," Indonesian Journal of Electrical Engineering and Computer Science,vol. 19, no. 1, pp. $248-256,2020$.

[26] A. Rahman, "Mobile edge computing for internet of things (IoT): security and privacy issues," Indones. J. Electr. Eng. Comput. Sci., vol. 18, no. 3, pp. 1486, 2020. 